\title{
Suppression of intrinsic roughness in encapsulated graphene
}

Thomsen, Joachim Dahl; Gunst, Tue; Gregersen, Søren Schou; Gammelgaard, Lene; Jessen, Bjarke Sørensen; Mackenzie, David; Watanabe, Kenji ; Taniguchi, Takashi ; Bøggild, Peter; Booth, Tim

Published in:

Physical Review B (Condensed Matter and Materials Physics)

Link to article, DOI:

10.1103/PhysRevB.96.014101

Publication date:

2017

Document Version

Publisher's PDF, also known as Version of record

Link back to DTU Orbit

Citation (APA):

Thomsen, J. D., Gunst, T., Gregersen, S. S., Gammelgaard, L., Jessen, B. S., Mackenzie, D., Watanabe, K., Taniguchi, T., Bøggild, P., \& Booth, T. (2017). Suppression of intrinsic roughness in encapsulated graphene. Physical Review B (Condensed Matter and Materials Physics), 96, [014101].

https://doi.org/10.1103/PhysRevB.96.014101

\section{General rights}

Copyright and moral rights for the publications made accessible in the public portal are retained by the authors and/or other copyright owners and it is a condition of accessing publications that users recognise and abide by the legal requirements associated with these rights.

- Users may download and print one copy of any publication from the public portal for the purpose of private study or research.

- You may not further distribute the material or use it for any profit-making activity or commercial gain

- You may freely distribute the URL identifying the publication in the public portal 


\title{
PHYSICAL REVIEW B 96, 014101 (2017) \\ cos \\ Suppression of intrinsic roughness in encapsulated graphene
}

\author{
Joachim Dahl Thomsen, ${ }^{1}$ Tue Gunst, ${ }^{1}$ Søren Schou Gregersen, ${ }^{1}$ Lene Gammelgaard, ${ }^{1}$ Bjarke Sørensen Jessen, ${ }^{1}$ \\ David M. A. Mackenzie, ${ }^{1}$ Kenji Watanabe, ${ }^{2}$ Takashi Taniguchi, ${ }^{2}$ Peter Bøggild,${ }^{1}$ and Timothy J. Booth ${ }^{1}$ \\ ${ }^{1}$ Center for Nanostructured Graphene, Department of Micro- and Nanotechnology, Technical University of Denmark, \\ DK-2800 Kongens Lyngby, Denmark \\ ${ }^{2}$ National Institute for Materials Science, 1-1 Namiki, Tsukuba 305-0044, Japan
}

(Received 21 March 2017; published 5 July 2017)

\begin{abstract}
Roughness in graphene is known to contribute to scattering effects which lower carrier mobility. Encapsulating graphene in hexagonal boron nitride $(\mathrm{hBN})$ leads to a significant reduction in roughness and has become the de facto standard method for producing high-quality graphene devices. We have fabricated graphene samples encapsulated by hBN that are suspended over apertures in a substrate and used noncontact electron diffraction measurements in a transmission electron microscope to measure the roughness of encapsulated graphene inside such structures. We furthermore compare the roughness of these samples to suspended bare graphene and suspended graphene on hBN. The suspended heterostructures display a root mean square (rms) roughness down to $12 \mathrm{pm}$, considerably less than that previously reported for both suspended graphene and graphene on any substrate and identical within experimental error to the rms vibrational amplitudes of carbon atoms in bulk graphite. Our first-principles calculations of the phonon bands in graphene/hBN heterostructures show that the flexural acoustic phonon mode is localized predominantly in the hBN layer. Consequently, the flexural displacement of the atoms in the graphene layer is strongly suppressed when it is supported by hBN, and this effect increases when graphene is fully encapsulated.
\end{abstract}

DOI: 10.1103/PhysRevB.96.014101

\section{INTRODUCTION}

Graphene is an atomically thin layer of $s p^{2}$-bonded carbon which displays substrate-induced roughness as a result of conforming to a surface [1], for example, silicon dioxide, and in the absence of a substrate has been shown to exhibit intrinsic ripples or corrugations [2]. Such ripples are posited as stabilizing long-range crystal order in graphene against thermodynamic instability (although practically achievable sample sizes are much smaller than where such instability becomes relevant) $[3,4]$ and are the origin of the negative thermal expansion coefficient of graphene [5], as well as broken $A-B$ symmetry [6]. Either form of roughness, whether intrinsic or imposed by a substrate, places an upper limit on the carrier mobility of graphene-based devices [7-9]. The suppression of roughness is therefore important for both technological applications that depend on the superior electrical transport properties of graphene and fundamental studies.

Suspending graphene has been shown to eliminate substrate-induced roughness and reduce the influence of other types of scattering, resulting in an order of magnitude improvement in carrier mobility to $200000 \mathrm{~cm}^{2} / \mathrm{V} \mathrm{s}$ at low temperatures [10] versus graphene supported on a substrate [11]. Subsequent work showing a similar order-ofmagnitude improvement in more mechanically robust samples of graphene encapsulated in hexagonal boron nitride (hBN) supported by $\mathrm{SiO}_{2}$ substrates is now the basis for routine production of high-performance graphene devices [12-15].

Pioneering work on the elimination of intrinsic and imposed roughness in graphene by using atomically flat mica substrates as a replacement for $\mathrm{SiO}_{2}$ showed a reduction in surface height variation from $1.2 \mathrm{~nm}$ to $25 \mathrm{pm}$ [16]. Roughness here was assessed by atomic force microscopy in noncontact mode to minimize the tip-sample interaction. Scanning tunneling microscopy work identifying the moiré pattern in graphene supported by hBN has shown a root-mean-square roughness $R_{\text {rms }}$ of 30.2 pm [17]; however, typically observed roughnesses of graphene on hBN assessed by antiferromagnetism [12] are significantly higher at around $100 \mathrm{pm}$, with this observed minimum achieved for $\mathrm{hBN}$ layers $17 \mathrm{~nm}$ or thicker, with similar values for such roughness reported in other works $[18,19]$.

Here, we present measurements of $R_{\text {rms }}$ of suspended graphene either freestanding, supported by hBN, or fully encapsulated on both sides by $\mathrm{hBN}$, performed using a diffraction tilt analysis in the transmission electron microscope (TEM) $[2,20]$. In contrast to scanning probe techniques, this method enables us to directly measure the roughness of graphene sheets within the bulk of a van der Waals heterostructure. We find $R_{\mathrm{rms}}$ of $114 \pm 1,21 \pm 2$, and $12 \pm 5 \mathrm{pm}$ for graphene freestanding, supported by hBN, and fully encapsulated, respectively. The $12 \pm 5 \mathrm{pm} R_{\mathrm{rms}}$ value is, to our knowledge, the lowest observed to date for graphene, with the roughness of a graphene sheet within a bulk heterostructure measured directly here. This value corresponds within experimental accuracy to the vibrational amplitude of carbon atoms in bulk graphite [21-23]. Using density functional theory (DFT) calculations of the hybridized phonon modes in graphene on monolayer $\mathrm{hBN}$ and graphene encapsulated by monolayer hBN, we show that the atomic displacements in graphene originate in the lowest-frequency gapped flexural phonon branch and that these decrease when graphene is fully encapsulated by $\mathrm{hBN}$. We find this trend consistent with our experimentally measured results. A strong localization of the lowest-frequency modes in the encapsulating $\mathrm{hBN}$ results in flexural displacements that are several orders of magnitude lower in the graphene layer.

\section{EXPERIMENTAL TECHNIQUES}

\section{A. Diffraction analysis of roughness}

The full three-dimensional inverse lattice of perfectly flat graphene is a set of $\delta$ functions [2]. Deviations of the lattice 

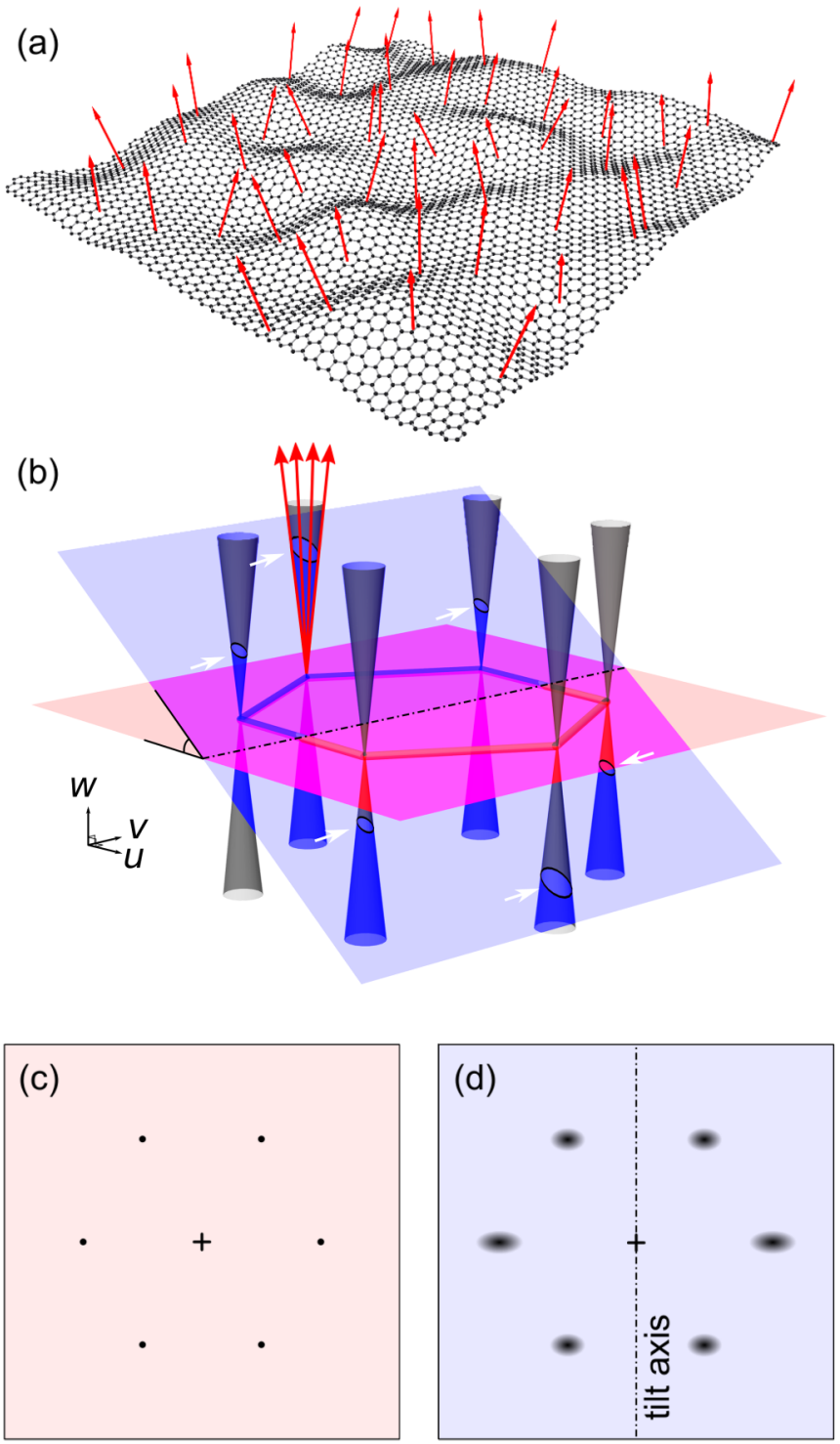

FIG. 1. (a) Schematic of ripples in graphene with normal vectors to the graphene lattice indicated (not to scale). (b) The reciprocal lattice of rippled graphene is a set of cones due to the angular distribution of real-space lattice normal vectors. Also shown are two planes which approximate the surface of the Ewald sphere, indicating the points of intersection with the reciprocal space lattice to form the diffraction pattern. The red plane shows the intersection at zero sample tilt, while the blue plane shows the intersection at a nonzero tilt angle. This leads to two distinctly different diffraction patterns: (c) one for zero tilt and (d) one for a tilted sample where the spots have become diffuse and the pattern has been stretched along an axis perpendicular to the tilt axis.

atoms from their in-plane positions will give rise to a collection of normal vectors pointing in different directions [Fig. 1(a)], turning these $\delta$ functions into diffuse cones in reciprocal space. The intersection of the Ewald sphere with the reciprocal lattice shows the points in reciprocal space which satisfy the Bragg condition and result in the measured diffraction pattern. The Ewald sphere can, in our case, be approximated by a plane since the diameter $d=1 / \lambda$, where $\lambda$ is the electron de Broglie wavelength, is very large compared to the reciprocal space lattice. The diffraction pattern is therefore the two-dimensional cut that the Ewald sphere makes with the reciprocal lattice. Figure 1(b) shows the reciprocal lattice for rippled graphene and the Ewald sphere at normal electron incidence (red plane), leading to the schematic diffraction pattern shown in Fig. 1(c). If the sample is tilted, the Ewald sphere intersects the cones at a certain height in the $w$ axis [Fig. 1(b), blue plane], resulting in blurring of the diffraction points and stretching of the overall pattern perpendicular to the tilt axis, as shown schematically in Fig. 1(d). In addition, the maximum intensity of the diffraction spot decreases due to this blurring. Both effects can be measured and provide a quantitative measure of the sample roughness.

$R_{\mathrm{rms}}$ is given by [20]

$$
R_{\mathrm{rms}}=\sqrt{\left\langle h^{2}\right\rangle}=\frac{1}{2 \pi} \sqrt{\frac{d \ln (I)}{d G^{2}}},
$$

where the distance of a diffraction spot in reciprocal space from the zero-order position is denoted $G$ and the maximum intensity of the diffraction spot is denoted $I$. See the Supplemental Material for a full derivation [24].

It follows that a measure for $R_{\text {rms }}$ can be extracted from the slope of $\ln (I)$ vs $G^{2}$. This method is applicable to both fully suspended and supported graphene on substrates, as long as the graphene diffraction spots are not obscured by the substrate diffraction pattern or depopulated by excess inelastic scattering from thick or dense substrates. Any potential attenuation of the graphene diffractions spots is also mitigated in this analysis by the fact that the roughness measurement depends solely on the gradient $\frac{d \ln (I)}{d G^{2}}$ and not the magnitude of the intensity itself.

In the following experiments, the aperture used for obtaining selective area electron diffraction patterns has a projected diameter on the sample of $184 \mathrm{~nm}$. For heterostructures and suspended graphene samples the exposure time used to obtain diffraction patterns was $20 \mathrm{~s}$. First-order spots farthest from the tilt axis are measured to minimize the errors in the determination in $G$ and $A$ variations since these spots vary the most with tilt. $G$ is measured as half the distance between a spot and its complementary spot on the other side of the tilt axis. A two-dimensional (2D) Gaussian distribution plus a linear background is fitted to the spot intensity by nonlinear regression, providing the maximum intensity, the major and minor axes of the distribution, and the angle of these axes with respect to the $u$ and $v$ axes in reciprocal space.

\section{B. Sample fabrication}

For full details of sample fabrication see the Supplemental Material [24]. Briefly, we have fabricated suspended graphene samples, suspended graphene on hBN, and suspended graphene encapsulated in $\mathrm{hBN}$ and placed these on TEM sample carrier chips capable of heating (DENS NanoChip XT) for TEM diffraction analysis. The heating function enables us to clean the samples in situ; see the next section.

\section{In situ cleaning of graphene}

Graphene and other 2D materials typically display adlayers of hydrocarbon and polymer contamination associated with resist residues that remain from transfer processes and 

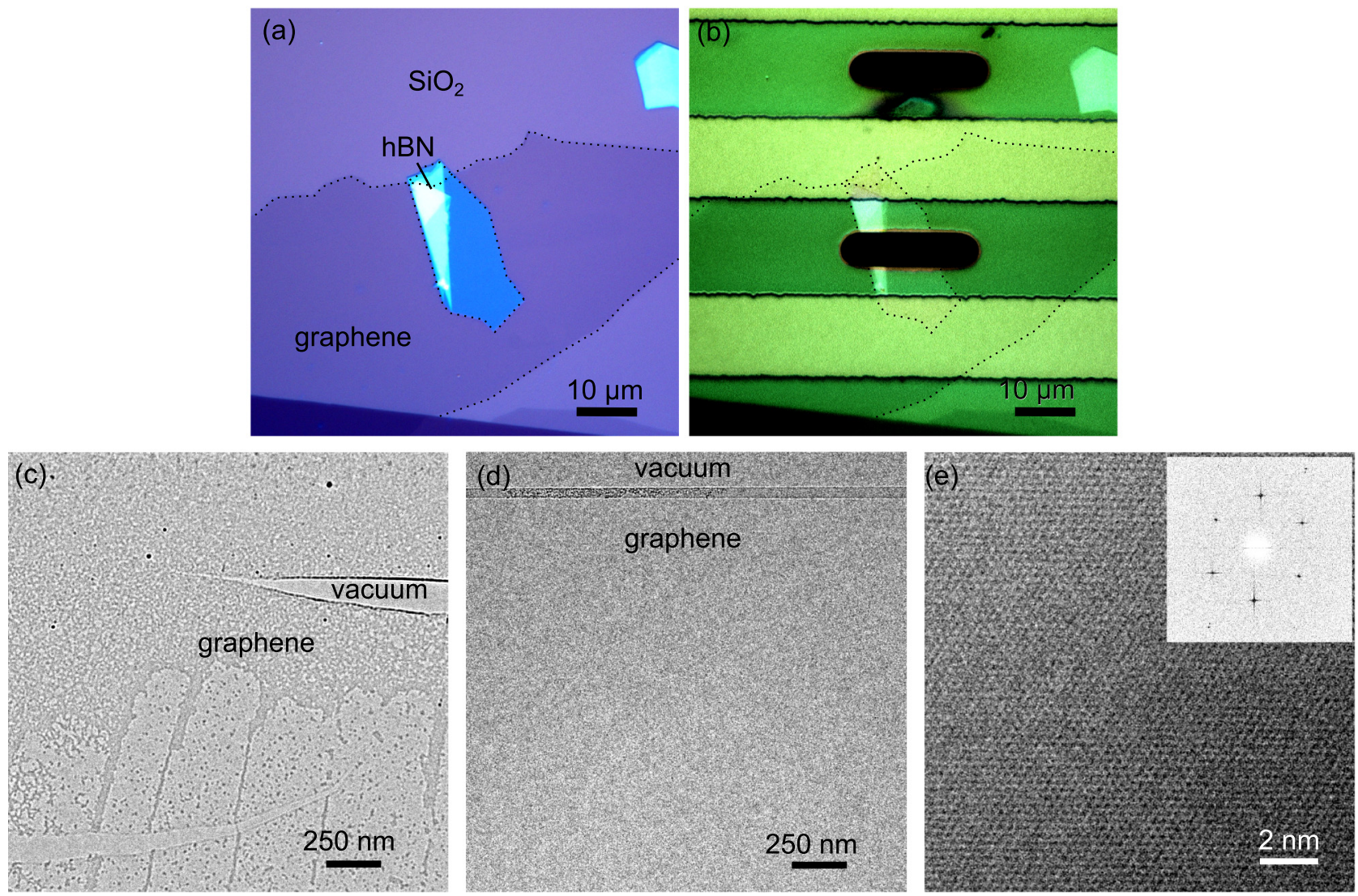

FIG. 2. (a) Optical image of a hBN flake on a graphene flake and (b) the same heterostructure as shown in (a) transferred to a TEM sample carrier. (c)-(e) TEM images of suspended graphene: (c) As-transferred graphene. (d) TEM image of annealed graphene showing no residual polymer contamination. A rolled graphene edge separates the graphene from vacuum and provides contrast in the absence of visible resist residues. (e) High-resolution image of the sample shown in (b); inset: fast Fourier transform of the main image. Both the first-order $2.1 \AA$ periodicity and a subset of the second-order $1.42 \AA$ periodicity from the graphene lattice are visible.

exposure to ambient conditions. Such adlayers are clearly visible as amorphous carbonaceous layers in the TEM and are difficult to remove. Typical cleaning procedures result in clean graphene patches of only nanometer dimensions [25-28]. Polymethyl methacrylate (PMMA) is often used as an intermediate supporting polymer during 2D materials transfer; however, PMMA residues in particular have proven to be challenging to remove by typical cleaning procedures such as thermal annealing in $\mathrm{Ar} / \mathrm{H}_{2}$ [23], and until now prevention strategies have proven to be the most effective cure [29].

Here, we instead use cellulose acetate butyrate (CAB) to transfer our graphene and hBN/graphene heterostructures. We find that $\mathrm{CAB}$ residues are readily removed by highvacuum annealing in the TEM $\left(500^{\circ} \mathrm{C}, 1 \times 10^{-7}\right.$ mbar, $\left.1 \mathrm{~h}\right)$. Importantly, before annealing we avoid exposing the sample to the electron beam, as this renders the annealing process ineffective in our experience, most likely due to cross-linking. This procedure results in the total cleaning of polymer residues from the entire suspended sample region (tens of micrometers across), save for a very small minority of isolated 10-100 nm-dimension-sized patches of residue which likely represent nonpolymeric or pre-cross-linked contaminants in the polymer. Figure 2(c) is a TEM image of as-transferred graphene showing polymeric contamination consisting of CAB residues, while Fig. 2(d) shows graphene which has been subjected to high-vacuum annealing as described above. Figure 2(e) is a high-resolution image of a region of the sample shown in Fig. 2(d), with a fast Fourier transform inset showing the expected lattice periodicity. The complete removal of polymeric residues across the images is apparent; however, this process also removes the largest sources of observable contrast in the images at lower magnifications, and high-resolution or diffraction imaging must be used to confirm the presence of graphene.

\section{EXPERIMENTAL RESULTS}

Typical diffraction patterns for graphene and hBN/graphene heterostructures at $0^{\circ}, 18^{\circ}$, and $36^{\circ}$ are shown in Fig. 3(a). We note that $G$ increases with tilt angle since the projected bond lengths decrease in real space. For graphene we see that the spots broaden and have significantly less intensity when tilted [see Fig. 3(b)]. For hBN on graphene the spots retain much of their intensity with tilt, already qualitatively showing a suppression of the roughness for graphene/hBN heterostructures.

In total one hBN encapsulated graphene sample, four hBN supported graphene samples, and three suspended graphene samples were characterized. The data shown in Fig. 3 were obtained from a single graphene flake which was partially 
(a)
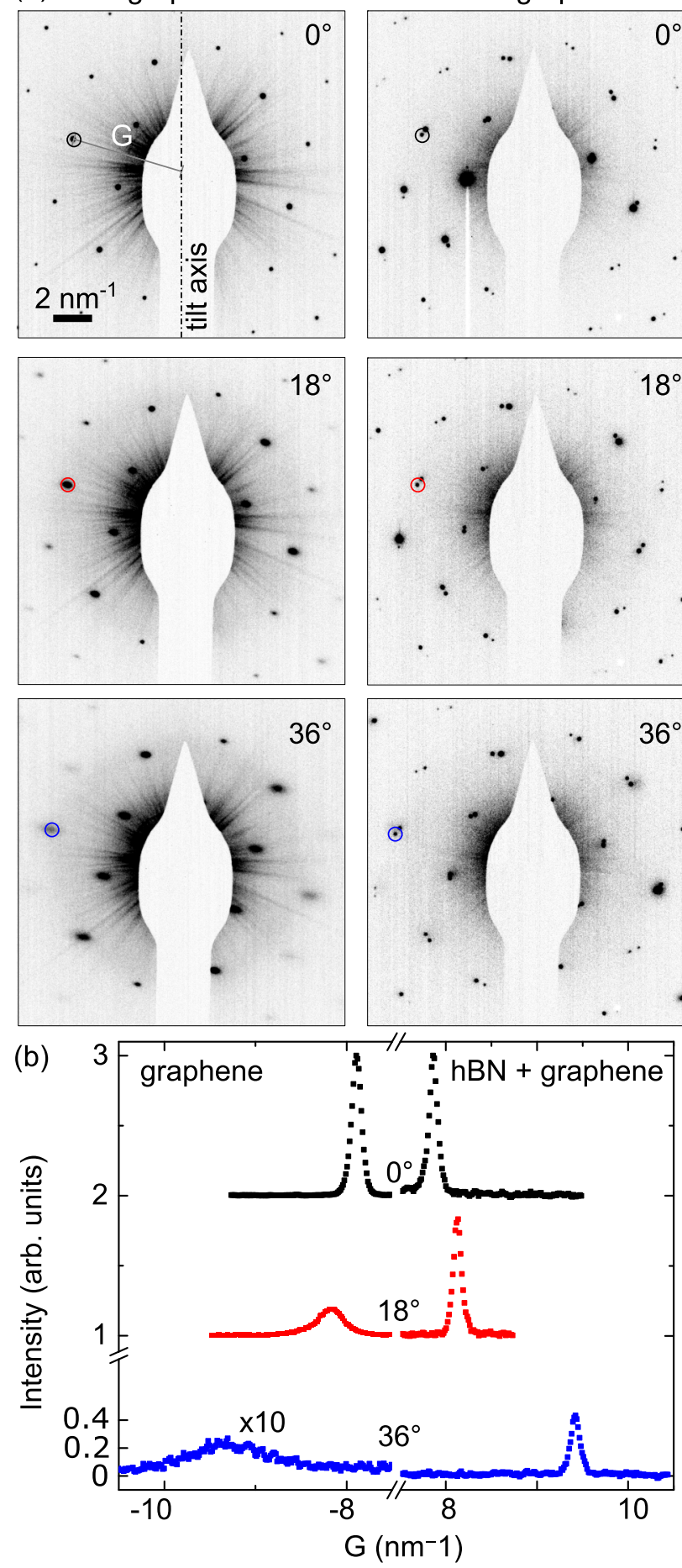

FIG. 3. (a) Diffraction patterns of graphene and a graphene/hBN heterostructure at $0^{\circ}, 18^{\circ}$, and $36^{\circ}$ tilt. (b) Intensity plots of the secondorder graphene diffraction spots circled in (a); note that the intensity is $10 \times$ for suspended graphene at $36^{\circ}$ tilt due to a very diffuse spot. Peaks are normalized to the $0^{\circ}$ tilt intensity.

supported by hBN and partially suspended. This allowed for the roughness values of suspended graphene and graphene supported by hBN to be compared within the same graphene flake. The resulting roughness measurements are plotted in Fig. 4 for the three different types of samples, where we plot

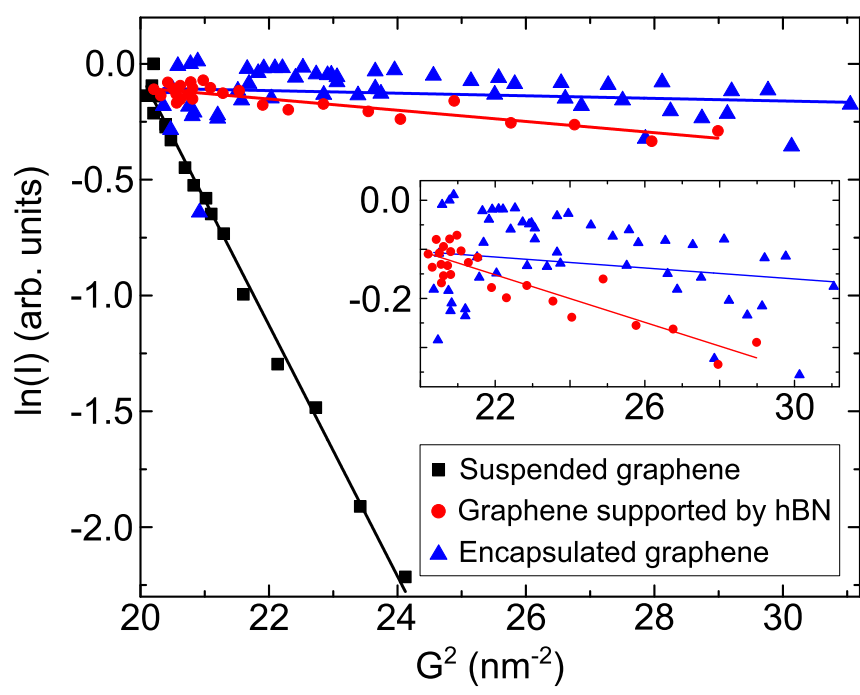

FIG. 4. Roughness measurements of a suspended graphene sample (black squares), graphene supported by hBN (red squares), and fully encapsulated graphene (blue triangles). The inset is a close-up of the data points for the graphene supported by $\mathrm{hBN}$ and fully encapsulated graphene. The solid lines are linear fits to the data points.

the logarithm of the maximum diffraction spot intensity versus $G^{2}$. For suspended bare graphene samples the diffraction spots quickly lose intensity with $G^{2}$. For graphene supported by hBN and for fully hBN encapsulated graphene the spot intensity decreases more slowly with tilt, with little deviation from the zero-tilt value. Linear regression is performed only in the range of $G^{2}$ values over which $2 \pi w h \leqslant 1.5$, where the slope of $\ln (I)$ vs. $G^{2}$ is constant to a good approximation (see Supplemental Material [24]).

The measured $R_{\mathrm{rms}}$ of the suspended graphene samples are $110 \pm 2,116 \pm 3$, and $117 \pm 1 \mathrm{pm}$. Graphene supported by $\mathrm{hBN}$ shows a greatly reduced $R_{\mathrm{rms}}$ of $19 \pm 4,25 \pm 1,19 \pm 3$, and $21 \pm 5 \mathrm{pm}$. Fully encapsulated graphene shows the lowest $R_{\text {rms }}$ of all the measured samples at $12 \pm 5 \mathrm{pm}$.

\section{CALCULATIONS OF ATOMIC DISPLACEMENTS}

\section{A. Phonon modes in heterostructures}

Previous theoretical studies have mainly focused on vibrations in free-standing graphene [30,31] and graphene on bulk substrates [32-34] while the important case of interaction between graphene and few layer hBN encapsulation is less well described [35]. As we will argue below the decreased roughness in encapsulated graphene can be ascribed to (i) the splitting of modes localized in the graphene and $\mathrm{hBN}$ layers and (ii) the renormalization of the bands when increasing the number of layers. The first principles methods and computational details are equivalent to those used in Ref. [36].

In Figs. 5(a) and 5(b), we show the calculated phonon dispersion and modes, respectively, when stacking monolayer graphene and an hBN monolayer. Consistent with previous calculations [35], the bands corresponding to in-plane modes are largely a superposition of the graphene and $\mathrm{hBN}$ phonon 

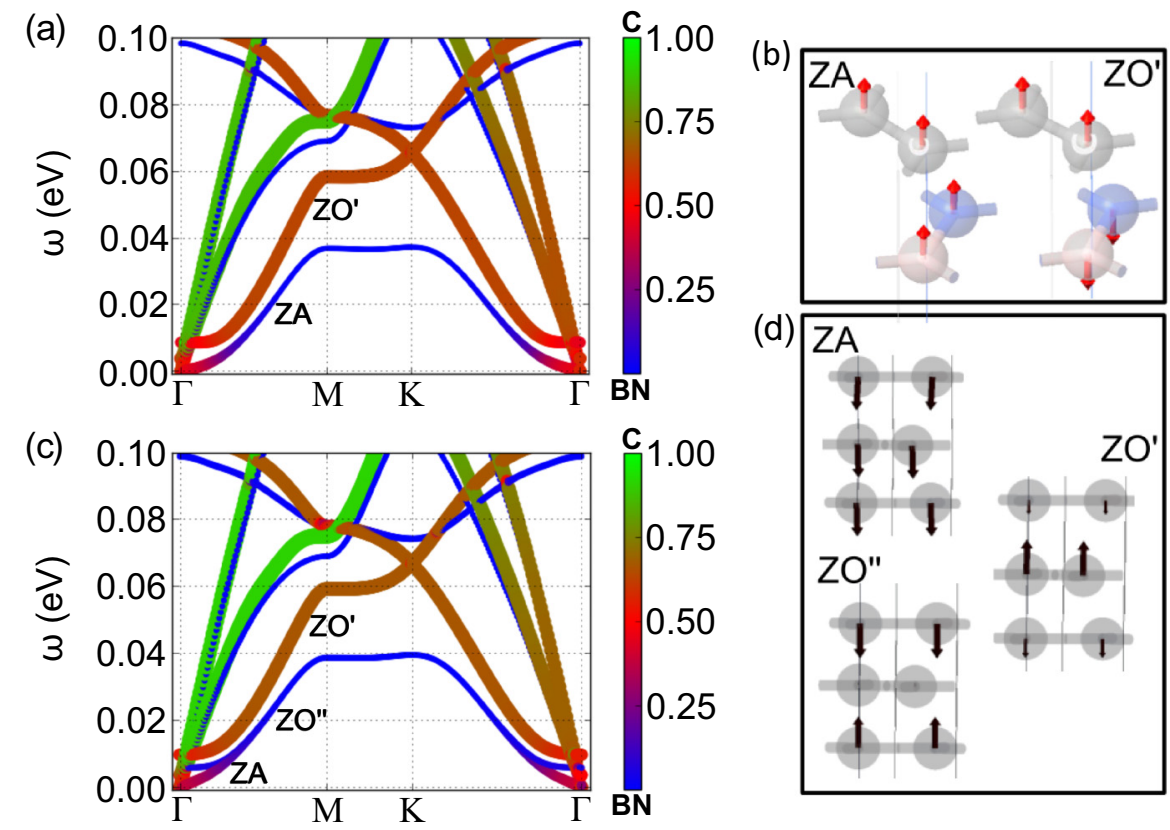

(d) ZA
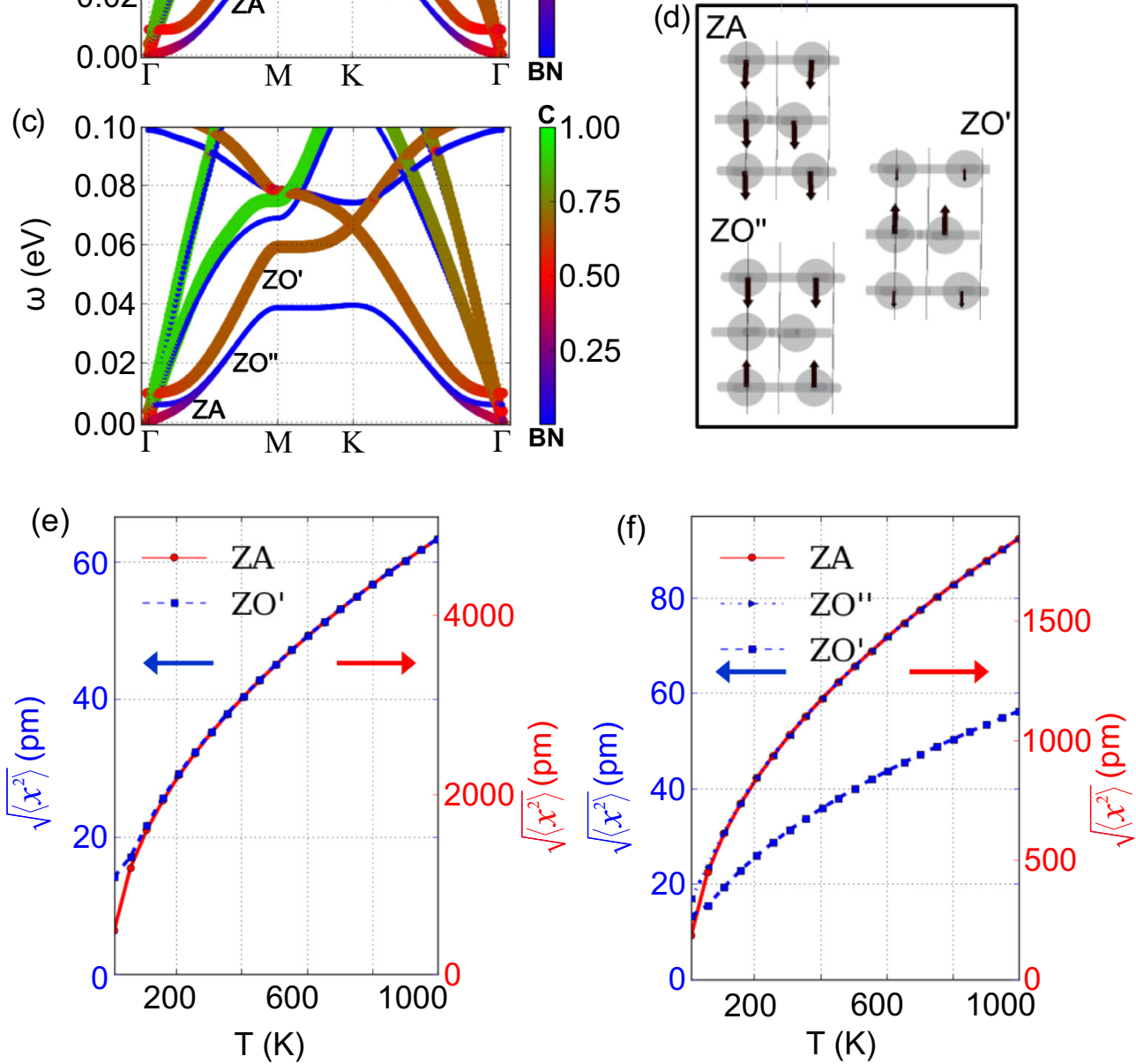

FIG. 5. (a) Phonon dispersion of $\mathrm{AB}$ stacking of graphene on monolayer hBN, with a $\mathrm{C}$ atom on top of a B atom. (c) $\mathrm{ABA}^{\prime}$ stacking of graphene fully encapsulated in monolayer hBN, with a $\mathrm{C}$ atom in between $\mathrm{B}$ and $\mathrm{N}$ atoms. (b) and (d) The labeled phonon modes are illustrated for $q=\Gamma$. (e) and (f) The rms displacement versus temperature for the flexural vibrations of (e) graphene on a monolayer of hBN and (f) graphene fully encapsulated in monolayer hBN.

dispersions. The two acoustic flexural (ZA) modes of the individual layers hybridize into a gapped mode $\left(\mathrm{ZO}^{\prime}\right)$ and a nongapped mode (ZA) with quadratic dispersion where atoms in graphene and hBN move out of phase and in phase in the $z$ direction, respectively [Fig. 5(b)]. If the hBN layer is completely clamped, only the gapped $\mathrm{ZO}^{\prime}$ mode exists (not shown). This indicates that the ZA mode is predominately localized in the hBN layer. In Figs. 5(a) and 5(c), we color the dispersion according to the contribution from each layer to the phonon mode to illustrate the localization of the flexural modes. Near the high-symmetry $q=\Gamma$ point the ZA motion hybridizes between the graphene and hBN layers. Away from the $\Gamma$ point this partition rapidly decreases from a mixed motion to a motion exclusively in the $\mathrm{hBN}$ layers. This indicates that mainly the $\mathrm{hBN}$ layer participates in the motion of the ZA mode. Consequently, the flexural displacement is expected to be larger in the hBN layer and smaller in the graphene layer, which mainly gets a contribution from the gapped $\mathrm{ZO}^{\prime}$ mode.

In the case of graphene encapsulated between two layers of monolayer hBN, illustrated in Figs. 5(c) and 5(d), we find three flexural acoustic modes, which we label ZA, ZO', and $\mathrm{ZO}^{\prime}$. The gap of the original $\mathrm{ZO}^{\prime}$ mode increases from a value of $8.55 \mathrm{meV}$ to a value of $9.66 \mathrm{meV}$, while an extra $\mathrm{ZO}^{\prime \prime}$ mode with a gap of $5.78 \mathrm{meV}$ emerges. Similar $\mathrm{ZO}^{\prime}$ gaps have been found in bilayer graphene $(10 \mathrm{meV})$ [37] and on graphene on $\mathrm{Cu} / \mathrm{sapphire}(6 \mathrm{meV})[38,39]$ and are a general feature of graphene on weakly interacting substrates $[34,35,40]$. The new $\mathrm{ZO}^{\prime \prime}$ mode is exclusively localized in the $\mathrm{hBN}$ layers. This is also shown in Fig. 5(d), illustrating the atomic motion near $\Gamma$. In addition, displacements in the graphene layer in the ZA mode are further decreased when an additional hBN layer is 
present, so that the flexural displacement of the encapsulated graphene is even lower.

We can evaluate the harmonic oscillator mean-square amplitude at a finite temperature from the obtained phonon dispersions and modes. We have that

$$
\left\langle\left(x_{\mathbf{q}}^{\lambda}\right)^{2}\right\rangle=\left(l_{\mathbf{q}}^{\lambda}\right)^{2} \operatorname{coth}\left(\frac{\hbar \omega_{\mathbf{q}}^{\lambda}}{2 k_{B} T}\right)
$$

where $\lambda$ labels the phonon mode, $\hbar$ is the reduced Planck constant, $\omega_{\mathbf{q}}^{\lambda}$ is the phonon frequency as a function of phonon momentum vector $\mathbf{q}, k_{B}$ is the Boltzmann constant, and $T$ is the temperature. The characteristic length of the harmonic oscillator is defined as

$$
l_{\mathbf{q}}^{\lambda}=\sqrt{\frac{\hbar}{2 \omega_{\mathbf{q}}^{\lambda} e_{\mathbf{q}}^{\lambda \dagger} m e_{\mathbf{q}}^{\lambda}}},
$$

where $e_{\mathbf{q}}^{\lambda}$ is the eigenmode of the vibration and $m$ is the diagonal mass matrix. The mean-square amplitude of a single atom can be obtained from the mode displacement:

$$
u_{\alpha \kappa}^{2}=\sum_{\lambda, \mathbf{q}}\left|e_{q}^{\lambda}\right|^{2}\left\langle\left(x_{q}^{\lambda}\right)^{2}\right\rangle .
$$

Here $\alpha$ labels a Cartesian coordinate of an atom labeled $\kappa . R_{\mathrm{rms}}$ of a given phonon mode is then defined as $R_{\mathrm{rms}}^{\lambda}=\sqrt{\left\langle\left(x_{q}^{\lambda}\right)^{2}\right\rangle}$. We find that the height fluctuations of the carbon atoms and $\mathrm{BN}$ atoms are equal to $R_{\mathrm{rms}}^{\mathrm{ZO}}$ and $R_{\mathrm{rms}}^{\mathrm{ZA}}$, respectively, as anticipated from the projected band structures in Figs. 5(a) and 5(b).

In Figs. 5(e) and 5(f), we demonstrate that the flexural displacement is far lower for the $\mathrm{ZO}^{\prime}$ and $\mathrm{ZO}^{\prime \prime}$ modes compared to the ZA modes. The displacement of the $\mathrm{ZO}^{\prime}$ and $\mathrm{ZO}^{\prime \prime}$ modes is almost independent of wavelength. For the ZA-mode displacement we choose a long-wavelength cutoff of $q_{c}=0.01$ in units of $\frac{2 \pi}{a}$ to mimic anharmonic long-wavelength renormalization. For comparison we obtain mean-square amplitudes for free-standing graphene and bulk graphite of 3000 and $30 \mathrm{pm}$, respectively.

For graphene supported by monolayer $\mathrm{hBN}$ at $300 \mathrm{~K}$, we obtain $R_{\mathrm{rms}}^{\mathrm{ZA}} \approx 2650 \mathrm{pm}$, while $R_{\mathrm{rms}}^{\mathrm{ZO}} \approx 35 \mathrm{pm}$. For graphene fully encapsulated in monolayer $\mathrm{hBN}$, we obtain a significantly lower $R_{\mathrm{rms}}^{\mathrm{ZA}} \approx 1010 \mathrm{pm}$ and $R_{\mathrm{rms}}^{\mathrm{ZO}} \approx 30 \mathrm{pm}$ and $\mathrm{RMS}^{\mathrm{ZO}} \approx$ $51 \mathrm{pm}$. Since the reduced roughness could be ascribed to a thicker sample, using the same analysis, we also considered graphene on top of two layers of $\mathrm{hBN}$. In this case we find $R_{\mathrm{rms}}^{\mathrm{ZA}} \approx 1327 \mathrm{pm}$ and $R_{\mathrm{rms}}^{\mathrm{ZO}} \approx 32 \mathrm{pm}$, highlighting a stronger impact on flexural displacements by the encapsulation compared to the effect of an additional layer. The roughness is therefore greatly reduced to values close to that of bulk graphite by encapsulating graphene with a few layers of hBN. Stacking or encapsulating graphene with hBN localizes the ZA mode predominately in the $\mathrm{hBN}$ layers. This explicitly means that the flexural displacement will be much lower for graphene on hBN and explains the increased flatness observed in the experiments.

\section{DISCUSSION}

Our roughness measurement of suspended graphene is lower than previously reported [20] and in excellent agreement with molecular dynamics simulations [41-43]. Tensile strain fields in graphene can suppress the anharmonic coupling between bending and stretching modes and lead to reduced roughness [44]. Hence, this difference could be caused by variations in roughness due to transfer-induced strain. Using Raman spectroscopy on separately prepared samples, we estimate the strain in our samples to be tensile with a value between $0.08 \%$ and $0.22 \%$, assuming purely biaxial and uniaxial strain, respectively (see Supplemental Material, Fig. S1 [24]), using published methods [45,46].

The hBN-supported graphene shows a much reduced roughness compared to previous reports of similar samples supported by $\mathrm{SiO}_{2}$ with an average $R_{\text {rms }}$ of $21 \pm 2 \mathrm{pm}$. This could suggest that while hBN is an excellent substrate for enhancing the flatness of graphene, $\mathrm{SiO}_{2}$ can still impose roughness through commonly used thicknesses of hBN and that for optimal results the $\mathrm{hBN}$-supported graphene structure should also ideally be suspended. The $12 \pm 5 \mathrm{pm} R_{\mathrm{rms}}$ measurement for fully encapsulated graphene is unique: the interior roughness of an encapsulated heterostructure is not accessible by scanning probe techniques. We note that the observed roughness is comparable within experimental error to the measured rms displacement of carbon atoms within bulk graphite [21-23]. This could be regarded as somewhat surprising, given that the stacking is performed manually and artificially and considering the lattice mismatch of graphene and hBN. Based on these results one would expect $A-B$ symmetry restoration and performance closer to ideal graphene devices, which would be a prime subject for transport studies in such heterostructures.

An additional confounding factor in experiments is possibly the differing thickness of $\mathrm{hBN}$ used in each case, which might affect the roughness. However, for the hBN-supported graphene we measured two samples with an hBN thickness of about $15 \mathrm{~nm}\left(R_{\mathrm{rms}}\right.$ of 19 and $\left.25 \mathrm{pm}\right)$ and two samples with hBN thickness of about $30 \mathrm{~nm}\left(R_{\mathrm{rms}}\right.$ of 18 and $\left.20 \mathrm{pm}\right)$ and found no significant effect of the hBN thickness. We also note that previous studies of the roughness of hBN supported by a substrate found the roughness to initially decrease with increasing hBN thickness, plateau, and reach a value of $100 \mathrm{pm}$ at about 17-nm hBN thickness [12]. Our hBN flakes have thicknesses within this plateau. The combined thickness of the fully encapsulated graphene sample was likewise about $30 \mathrm{~nm}$. We therefore do not expect the combined thickness of the sample to be the reason for the low $(12 \mathrm{pm}) R_{\mathrm{rms}}$. Finally, two different types of hBN were used for the hBN-supported graphene samples [47], again without significant difference in the measured $R_{\text {rms }}$.

In our simulations, graphene supported by monolayer hBN shows an rms carbon atom displacement of $35 \mathrm{pm}$, considerably higher than experiment $(21 \mathrm{pm})$. We do note, however, that this calculation was done for hBN monolayers, while hBN crystals of 15-30-nm thickness were used in experiments. The simulation of graphene on two hBN layers shows an rms carbon atom displacement of $32 \mathrm{pm}$. In this picture the $35-\mathrm{pm}$ $R_{\text {rms }}$ value represents an upper limit for the surface roughness 
of a suspended graphene on hBN sample, which should be reduced for thicker flakes. Graphene encapsulated in $\mathrm{hBN}$ monolayers shows the smallest roughness of $30 \mathrm{pm}$ with a discrepancy between measurement $\left(R_{\mathrm{rms}}\right.$ of $\left.12 \mathrm{pm}\right)$ likely due to similar reasons. In experiments we find that graphene on the surface of a heterostructure with $\mathrm{hBN}$ appears to have a larger roughness than graphene encapsulated in $\mathrm{hBN}$, irrespective of the hBN thickness used. This finding is supported by our simulations which show that encapsulating graphene in hBN monolayers suppresses flexural displacements more than the addition of another supporting hBN layer even if the total thickness of the system is the same.

\section{SUMMARY AND CONCLUSIONS}

We have used noncontact diffraction measurements using TEM to measure the roughness of a monolayer graphene encapsulated in hBN and observed the roughness in such heterostructures to be $12 \pm 5 \mathrm{pm}$, consistent with that of an individual graphene layer in bulk graphite within experimental error. In addition, we have performed the same analysis for hBN-supported graphene over an aperture and measured an $R_{\mathrm{rms}}$ of $21 \pm 2 \mathrm{pm}$. The graphene in such a heterostructure is flatter when suspended over an aperture than when supported by $\mathrm{SiO}_{2}$ since previous measurements show a roughness of 30-100 pm for $\mathrm{SiO}_{2}$-supported hBN/graphene. Finally, the measurements for suspended graphene show an $R_{\mathrm{rms}}$ of $114 \pm 1 \mathrm{pm}$, in good agreement with molecular dynamics simulations. Our first-principles calculations support the idea that the roughness of a graphene layer within such a heterostructure should be lower than would be the case for hBN-supported graphene due to an increased localization of the flexural acoustic phonon mode in the hBN layers when graphene is fully encapsulated.

Our results show that for the minimization of roughness, van der Waals heterostructures should be suspended even when the total thickness of the heterostructure is $30 \mathrm{~nm}$ or more since the substrate can still impose roughness. Since roughness places an upper limit for the carrier mobility in graphene devices, suspending devices in this way should provide the best performance. In addition, while the preparation of such samples is more complex than for substrate-supported samples, the additional thickness of such heterostructures makes them more robust than the corresponding unencapsulated samples in practical applications.

\section{ACKNOWLEDGMENTS}

The work leading to these results received funding from the European Union Seventh Framework Programme (FP/20072013) under Grant Agreement No. FP7-604000. The Center for Nanostructured Graphene is sponsored by the Danish National Research Foundation, Project No. DNRF103. T.G. acknowledges support from Innovation Fund Denmark (Grant No. 79-2013-1). K.W. and T.T. acknowledge support from the Elemental Strategy Initiative conducted by MEXT, Japan, and JSPS KAKENHI Grants No. JP26248061, No. JP15K21722, and No. JP25106006.
[1] M. Ishigami, J. H. Chen, W. G. Cullen, M. S. Fuhrer, and E. D. Williams, Nano Lett. 7, 1643 (2007).

[2] J. C. Meyer, A. K. Geim, M. I. Katsnelson, K. S. Novoselov, T. J. Booth, and S. Roth, Nature (London) 446, 60 (2007).

[3] N. D. Mermin, Phys. Rev. 176, 250 (1968).

[4] A. Fasolino, J. H. Los, and M. I. Katsnelson, Nat. Mater. 6, 858 (2007).

[5] P. L. de Andres, F. Guinea, and M. I. Katsnelson, Phys. Rev. B 86, 144103 (2012).

[6] J. Sánchez-Barriga, A. Varykhalov, D. Marchenko, M. R. Scholz, and O. Rader, Phys. Rev. B 85, 201413 (2012).

[7] A. H. Castro Neto, F. Guinea, N. M. R. Peres, K. S. Novoselov, and A. K. Geim, Rev. Mod. Phys. 81, 109 (2009).

[8] J.-H. Chen, C. Jang, S. Xiao, M. Ishigami, and M. S. Fuhrer, Nat. Nanotechnol. 3, 206 (2008).

[9] M. I. Katsnelson and A. K. Geim, Philos. Trans. R. Soc. A 366, 195 (2008).

[10] K. I. Bolotin, K. J. Sikes, Z. Jiang, M. Klima, G. Fudenberg, J. Hone, P. Kim, and H. L. Stormer, Solid State Commun. 146, 351 (2008).

[11] K. S. Novoselov, A. K. Geim, S. V. Morozov, D. Jiang, Y. Zhang, S. V. Dubonos, I. V. Grigorieva, and A. A. Firsov, Science 306, 666 (2004).

[12] C. R. Dean, A. F. Young, I. Meric, C. Lee, L. Wang, S. Sorgenfrei, K. Watanabe, T. Taniguchi, P. Kim, K. L. Shepard, and J. Hone, Nat. Nanotechnol. 5, 722 (2010).

[13] C. R. Dean, L. Wang, P. Maher, C. Forsythe, F. Ghahari, Y. Gao, J. Katoch, M. Ishigami, P. Moon, M. Koshino, T. Taniguchi, K.
Watanabe, K. L. Shepard, J. Hone, and P. Kim, Nature (London) 497, 598 (2013).

[14] L. Wang, I. Meric, P. Y. Huang, Q. Gao, Y. Gao, H. Tran, T. Taniguchi, K. Watanabe, L. M. Campos, D. A. Muller, J. Guo, P. Kim, J. Hone, K. L. Shepard, and C. R. Dean, Science 342, 614 (2013).

[15] F. Pizzocchero, L. Gammelgaard, B. S. Jessen, J. M. Caridad, L. Wang, J. Hone, P. Bøggild, and T. J. Booth, Nat. Commun. 7, 11894 (2016).

[16] C. H. Lui, L. Liu, K. F. Mak, G. W. Flynn, and T. F. Heinz, Nature (London) 462, 339 (2009).

[17] J. Xue, J. Sanchez-Yamagishi, D. Bulmash, P. Jacquod, A. Deshpande, K. Watanabe, T. Taniguchi, P. Jarillo-Herrero, and B. J. LeRoy, Nat. Mater. 10, 282 (2011).

[18] M. Yamamoto, T. L. Einstein, M. S. Fuhrer, and W. G. Cullen, ACS Nano 6, 8335 (2012).

[19] J. H. Lee, A. Avsar, J. Jung, J. Y. Tan, K. Watanabe, T. Taniguchi, S. Natarajan, G. Eda, S. Adam, A. H. Castro Neto, and B. Özyilmaz, Nano Lett. 15, 319 (2015).

[20] D. A. Kirilenko, A. T. Dideykin, and G. Van Tendeloo, Phys. Rev. B 84, 235417 (2011).

[21] R. Chen and P. Trucano, Acta Crystallogr. A 34, 979 (1978).

[22] E. Fitzer and U. Funk, Carbon 16, 85 (1978).

[23] B. Firey, F. W. de Wette, E. de Rouffignac, and G. P. Alldredge, Phys. Rev. B 28, 7210 (1983).

[24] See Supplemental Material at http://link.aps.org/supplemental/ 10.1103/PhysRevB.96.014101 for details on sample fabrication, strain estimation in suspended graphene samples, and the 
derivation of graphene roughness from tilted electron diffraction patterns. The Supplemental Material includes Refs. [48-50].

[25] Y.-C. Lin, C.-C. Lu, C.-H. Yeh, C. Jin, K. Suenaga, and P.-W. Chiu, Nano Lett. 12, 414 (2012).

[26] Y.-C. Lin, C. Jin, J.-C. Lee, S.-F. Jen, K. Suenaga, and P.-W. Chiu, ACS Nano 5, 2362 (2011).

[27] S. Liu, Q. Zhao, J. Xu, K. Yan, H. Peng, F. Yang, L. You, and D. Yu, Nanotechnology 23, 085301 (2012).

[28] B. Alemán, W. Regan, S. Aloni, V. Altoe, N. Alem, C. Girit, B. Geng, L. Maserati, M. Crommie, F. Wang, and A. Zettl, ACS Nano 4, 4762 (2010).

[29] Z. Li, A. Kozbial, N. Nioradze, D. Parobek, G. J. Shenoy, M. Salim, S. Amemiya, L. Li, and H. Liu, ACS Nano 10, 349 (2016).

[30] E. Mariani and F. von Oppen, Phys. Rev. Lett. 100, 076801 (2008).

[31] L. Lindsay, D. A. Broido, and N. Mingo, Phys. Rev. B 82, 115427 (2010).

[32] H. W. Kim, W. Ko, J. Ku, I. Jeon, D. Kim, H. Kwon, Y. Oh, S. Ryu, Y. Kuk, S. W. Hwang, and H. Suh, Nat. Commun. 6, 7528 (2015).

[33] Z.-Y. Ong and E. Pop, Phys. Rev. B 84, 075471 (2011).

[34] B. Amorim and F. Guinea, Phys. Rev. B 88, 115418 (2013).

[35] G. J. Slotman, G. A. de Wijs, A. Fasolino, and M. I. Katsnelson, Ann. Phys. (Berlin, Ger.) 526, 381 (2014).

[36] T. Gunst, T. Markussen, K. Stokbro, and M. Brandbyge, Phys. Rev. B 93, 035414 (2016).

[37] C. H. Lui, L. M. Malard, S. Kim, G. Lantz, F. E. Laverge, R. Saito, and T. F. Heinz, Nano Lett. 12, 5539 (2012).
[38] A. Al Taleb, H. K. Yu, G. Anemone, D. Farías, and A. M. Wodtke, Carbon 95, 731 (2015).

[39] G. Anemone, E. Climent-Pascual, H. K. Yu, A. Al Taleb, F. Jiménez-Villacorta, C. Prieto, A. M. Wodtke, A. De Andrés, and D. Farías, RSC Adv. 6, 21235 (2016).

[40] A. A. Taleb and D. Farías, J. Phys. Condens. Matter 28, 103005 (2016).

[41] S. K. Singh, M. Neek-Amal, S. Costamagna, and F. M. Peeters, Phys. Rev. B 87, 184106 (2013).

[42] W. Gao and R. Huang, J. Mech. Phys. Solids 66, 42 (2014).

[43] R. Ramírez, E. Chacón, and C. P. Herrero, Phys. Rev. B 93, 235419 (2016).

[44] R. Roldán, A. Fasolino, K. V. Zakharchenko, and M. I. Katsnelson, Phys. Rev. B 83, 174104 (2011).

[45] L. Banszerus, H. Janssen, M. Otto, A. Epping, T. Taniguchi, K. Watanabe, B. Beschoten, D. Neumaier, and C. Stampfer, 2D Mater. 4, 025030 (2017).

[46] J. E. Lee, G. Ahn, J. Shim, Y. S. Lee, and S. Ryu, Nat. Commun. 3, 1024 (2012).

[47] The hBN-supported graphene samples were purchased from HQgraphene and produced by K.W. and T.T.

[48] T. Taniguchi and K. Watanabe, J. Cryst. Growth 303, 525 (2007).

[49] L. Schué, I. Stenger, F. Fossard, A. Loiseau, and J. Barjon, 2D Mater. 4, 015028 (2016).

[50] G. F. Schneider, V. E. Calado, H. Zandbergen, L. M. K. Vandersypen, and C. Dekker, Nano Lett. 10, 1912 (2010). 